\title{
Fatores de risco associados ao desenvolvimento do pé diabético e ações executadas na Atenção Primária à Saúde para prevenção do agravo
}

\author{
Risk factors associated with the development of diabetic foot and actions performed in \\ Primary Health Care for disease prevention
}
Factores de riesgo asociados con el desarrollo del pie diabético y acciones realizadas en Atención Primaria de Salud para la prevención de la enfermedad

\begin{abstract}
Martha Teixeira do Nascimento ${ }^{1}$, Nayra Iolanda de Oliveira Silva ${ }^{1}$, Fabrícia Castelo Branco de Andrade Brito ${ }^{1,2}$, Francisco Lucas de Lima Fontes ${ }^{1 *}$, Ariane Freire Oliveira ${ }^{3}$, João Victor Alves Oliveira $^{3}$, Josélia Costa Soares ${ }^{1}$, Sâmara Gabriele Ferreira de Brito², Maria Oneide dos Santos ${ }^{1}$, Daniely Matias Facundes ${ }^{4}$, Ingrid Tainá Sousa Dias ${ }^{1}$, Regina Célia Soares de Sousa Ponciano ${ }^{5}$, Elis Jordana Crispim Alencar ${ }^{1}$, Samaira Ferreira de Lira ${ }^{1}$, Rosiane Ferreira da Luz ${ }^{6}$.
\end{abstract}

\section{RESUMO}

Objetivo: Identificar os fatores de risco associados ao desenvolvimento do pé diabético, bem como as ações executadas na Atenção Primária à Saúde para prevenção do agravo. Métodos: Trata-se de uma revisão integrativa de natureza exploratória, realizada por meio da biblioteca virtual Scientific Eletronic Library Online e nas bases de dados Banco de Dados de Enfermagem, Literatura Latino-Americana e do Caribe em Ciências da Saúde e Portal da CAPES, utilizando os descritores "pé diabético", "autocuidado", "assistência de Enfermagem", "atenção primária à saúde" e "enfermeiro". Foram selecionados vinte artigos para construção da discussão. Resultados: Os fatores de risco para o pé diabético relacionavam-se a déficits de conhecimento do paciente, baixa escolaridade, poder aquisitivo limitado, desconhecimento de comorbidades e não adesão ao tratamento. Esses fatores repercutem na adoção de práticas de autocuidado. As ações preventivas identificadas voltavam-se principalmente à execução da consulta de Enfermagem, com avaliação clínica e orientações; e práticas de educação em saúde. Conclusão: Percebe-se condutas direcionadas essencialmente a orientações com incentivo à obtenção de conhecimento acerca do processo saúde-doença. Compreende-se, portanto, que o entendimento sobre o estado de saúde empodera o diabético e estimula seu protagonismo e conscientização na adesão às práticas de autocuidado.

Palavras-chave: Pé diabético, Atenção Primária à Saúde, Assistência de Enfermagem.

\footnotetext{
ABSTRACT

Objective: To identify the risk factors associated with the development of diabetic foot, as well as the actions performed in Primary Health Care for disease prevention. Methods: This is an exploratory integrative review, conducted through the Scientific Electronic Library Online and in the Banco de Dados de Enfermagem, Literatura Latino-Americana e do Caribe em Ciências da Saúde and Portal da CAPES, using the keywords

${ }^{1}$ Faculdade UNINASSAU - Campus Redenção. Teresina, Piauí, Brasil. *E-mail: lucasfontesenf@hotmail.com

2 Universidade Federal do Piauí. Teresina, Piauí, Brasil.

${ }^{3}$ Centro Universitário UNINOVAFAPI. Teresina, Piauí, Brasil.

${ }^{4}$ Associação de Ensino Superior do Piauí. Teresina, Piauí, Brasil.

${ }^{5}$ Faculdade de Tecnologia e Educação Superior Profissional. Teresina, Piauí, Brasil.

${ }^{6}$ Centro Universitário Santo Agostinho. Teresina, Piauí, Brasil.
} 
"pé diabético", "autocuidado", "assistência de Enfermagem", "atenção primária à saúde" and "enfermeiro". Twenty articles were selected to build the discussion. Results: Risk factors for diabetic foot were related to patient knowledge deficits, low education, limited purchasing power, lack of comorbidities and non-adherence to treatment. These factors affect the adoption of self-care practices. The preventive actions identified mainly focused on the execution of the nursing consultation, with clinical evaluation and guidance; and health education practices. Conclusion: It is perceived conduct directed essentially to guidelines with incentive to obtain knowledge about the health-disease process. Therefore, it is understood that the understanding about health status empowers the diabetic and stimulates their protagonism and awareness in adherence to selfcare practices.

Keywords: Diabetic foot, Primary Health Care, Nursing care.

\section{RESUMEN}

Objetivo: Identificar los factores de riesgo asociados con el desarrollo del pie diabético, así como las acciones realizadas en Atención Primaria de Salud para la prevención del agravo. Métodos: Esta es una revisión exploratoria integradora, realizada a través de la biblioteca virtual en línea de la Scientific Electronic Library Online, Banco de Dados de Enfermagem, Literatura Latino-Americana e do Caribe em Ciências da Saúde y Portal da CAPES, utilizando las palabras clave "pé diabético", "autocuidado", "assistência de Enfermagem", "atenção primária à saúde" y "enfermeiro". Se seleccionaron veinte artículos para construir la discusión. Resultados: Los factores de riesgo para el pie diabético se relacionaron con déficits de conocimiento del paciente, baja educación, poder adquisitivo limitado, falta de comorbilidades y falta de adherencia al tratamiento. Estos factores afectan la adopción de prácticas de autocuidado. Las acciones preventivas identificadas se centraron principalmente en la ejecución de la consulta de enfermería, con evaluación clínica y orientación; y prácticas de educación en salud. Conclusión: Se percibe una conducta dirigida esencialmente a pautas con incentivos para obtener conocimiento sobre el proceso salud-enfermedad. Por lo tanto, se entiende que la comprensión del estado de salud empodera a los diabéticos y estimula su protagonismo y conciencia en la adhesión a las prácticas de autocuidado.

Palabras-clave: Pie diabético, Atención Primaria de Salud, Atención de Enfermería.

\section{INTRODUÇÃO}

Portadores de diabetes mellitus (DM) necessitam de acompanhamento por equipe multidisciplinar em saúde com fornecimento de recursos necessários para manutenção do autocuidado e prevenção de agravos. A doença caracteriza-se como uma disfunção metabólica crônica degenerativa evidenciada por hiperglicemia crônica, ocasionada pela destruição das células beta pancreáticas, resistência à ação e/ou mau funcionamento na secreção de insulina (OLIVEIRA PS et al., 2016).

Estima-se que mundialmente 415 milhões de pessoas sejam diabéticas. No Brasil, o número de casos novos elevou-se $61,8 \%$ entre o período de 2006 e 2016, taxa associada ao quantitativo de sujeitos que dizem ter conhecimento do diagnóstico de DM (BRASIL, 2016).

Por esses motivos é considerada um grave problema de saúde pública e de elevada prevalência mundialmente, ao trazer profundas repercussões na qualidade de vida do indivíduo, não somente por seus efeitos danosos e complicações, mas também pelas modificações no estilo de vida, necessárias ao controle da doença (PETERMANN XB et al., 2015).

É uma doença crônica não transmissível (DCNT) com elevada morbimortalidade resultante, frequentemente, da vascularização periférica ineficaz, aspecto este que provoca disfunções em diversos órgãos. Entre as complicações, encontram-se a neuropatia, nefropatia, macroangiopatias e a retinopatia, responsáveis por agravos como amputação não traumática de membros inferiores, doença renal crônica, doenças cardiovasculares e cegueira (OLIVEIRA PS et al., 2016). 
A úlcera de membros inferiores, conhecida popularmente como pé diabético, corresponde a uma das complicações mais graves da doença e pode progredir para amputações menores e maiores. Este agravo aumenta a mortalidade, modifica $\circ$ padrão de qualidade de vida, prolonga 0 tratamento hospitalar ou ambulatorial e repercute no absenteísmo laboral e aposentadorias prematuras (OLIVEIRA NETO M et al., 2017). Aproximadamente $15 \%$ das pessoas diabéticas progridem com úlceras nos pés, o que acarreta $85 \%$ de amputações não traumáticas em membros inferiores (SMANIOTO FN et al., 2014).

Na Atenção Primária à Saúde (APS) praticam-se ações voltadas ao indivíduo e ao grupo ao qual está inserido, destinando-se sanar os problemas habitualmente encontrados na comunidade, entre eles as DCNTs, as quais por envolverem causas múltiplas, requerem uma atenção integral. É o local ideal para acompanhamento do paciente diabético, por ser resolutiva à população que necessita de acesso a cuidados continuados durante toda a vida, também se ocupa de ações mais básicas, envolvendo dispensação de medicamentos, educação em saúde, aconselhamento e acompanhamento contínuo (BRASIL, 2016).

A prevenção do pé diabético deve ocorrer prioritariamente na APS, por profissionais devidamente capacitados para orientar, tendo esta estratégia como fundamental para a redução de possíveis complicações. Todo indivíduo diabético deve ser rastreado, reconhecendo aqueles predispostos ao desenvolvimento de ulcerações nos pés, podendo beneficiá-lo com intervenções profiláticas, sendo o estímulo ao autocuidado uma delas (SILVA CAM et al., 2014).

O enfermeiro possui atribuição relevante na avaliação sistemática dos pés e no reconhecimento dos fatores de risco em cada indivíduo, visando a queda do número de novos casos de pé diabético e amputações. Esse exame deve associar-se à história pregressa do paciente, averiguando lesões anteriores e amputações prévias, e identificação da habilidade ou não do diabético em instituir medidas de autocuidado com seus pés (IMAZU MFM et al., 2015).

Este estudo justifica-se por sua relevância acadêmica e social, ao promover reflexões sobre os obstáculos de autocuidado encarados pela pessoa diabética nos aspectos preventivos da complicação discutida. Além da importância de trazer à tona questões inerentes à saúde desse paciente na APS. A prevenção do pé diabético depende de inúmeros fatores como a qualidade do atendimento ofertado, satisfação do sujeito quanto à assistência, incentivo ao autocuidado e intenção do diabético em seguir orientações para a saúde. Faz-se necessário rastreamento, no âmbito da APS, daqueles com risco de evolução para este agravo e classificação dos que já possuem comprometimento neuropático. Este estudo poderá contribuir na melhora da qualidade de vida de pacientes em risco de desenvolvimento desta complicação, ao ressaltar a relevância de prevenção de uma das consequências da DM não tratada adequadamente.

Diante do que foi explanado, o objetivo deste artigo foi identificar os fatores de risco associados ao desenvolvimento do pé diabético, bem como as ações executadas na Atenção Primária à Saúde para prevenção do agravo.

\section{MÉTODOS}

Este estudo fundamenta-se em uma revisão integrativa de natureza exploratória. Para sua construção seguiram-se seis estágios: produção da hipótese ou questionamento norteador, pesquisa ou amostragem na literatura, coleta de dados, exame crítico dos estudos incluídos, discussão dos resultados e divulgação do estudo (ERCOLE FF et al., 2014).

O questionamento norteador foi preparado por meio do recurso PICO (P: paciente ou problema, I: intervenção, C: comparação e O: outcomes ou desfecho) segundo o qual surgiu: "Quais os fatores de risco associados ao desenvolvimento do pé diabético e as ações executadas no âmbito da Atenção Primária à Saúde para prevenção do agravo?"

O estudo foi executado nos meses de fevereiro a maio de 2019, empregando os seguintes métodos de inclusão: pesquisas disponíveis na íntegra, gratuitas, em língua portuguesa, publicados entre 2014 e 2018. Como métodos de exclusão definiu-se não fazer uso de livros, monografias, dissertações, teses, textos incompletos e estudos que não estavam associados ao tema central analisado. 
A busca pelos estudos na literatura ocorreu por intermédio de pesquisa na biblioteca virtual Scientific Eletronic Library Online (SciELO) e nas bases de dados Banco de Dados de Enfermagem (BDEnf), Literatura Latino-Americana e do Caribe em Ciências da Saúde (LILACS) e Portal da CAPES (Portal de Periódicos da Coordenação de Aperfeiçoamento de Pessoal de Nível Superior).

Para a coleta dos estudos, utilizaram-se os descritores "pé diabético", "autocuidado", "assistência de Enfermagem", "atenção primária à saúde" e "enfermeiro". Essas palavras-chave foram cruzadas aplicandose o recurso booleano "and", além do filtro de limites temporais estabelecido nos critérios de inclusão.

Finalizadas as buscas, encontrou-se uma amostra com 192 estudos importantes ao tema estudo, conforme esquema da Figura 1. Para execução deste estudo os artigos foram escolhidos e sujeitados a exame exploratório, analítico e interpretativo, dentre eles 20 estudos que mais estavam de acordo com tema foram empregados para construção desta revisão integrativa, sendo: 5 artigos da LILACS, 6 artigos da SciELO, 2 artigos do Portal da CAPES e 7 artigos do BDEnf.

Figura 1 - Processo de busca e seleção dos artigos. Teresina, Piauí, Brasil, 2019.

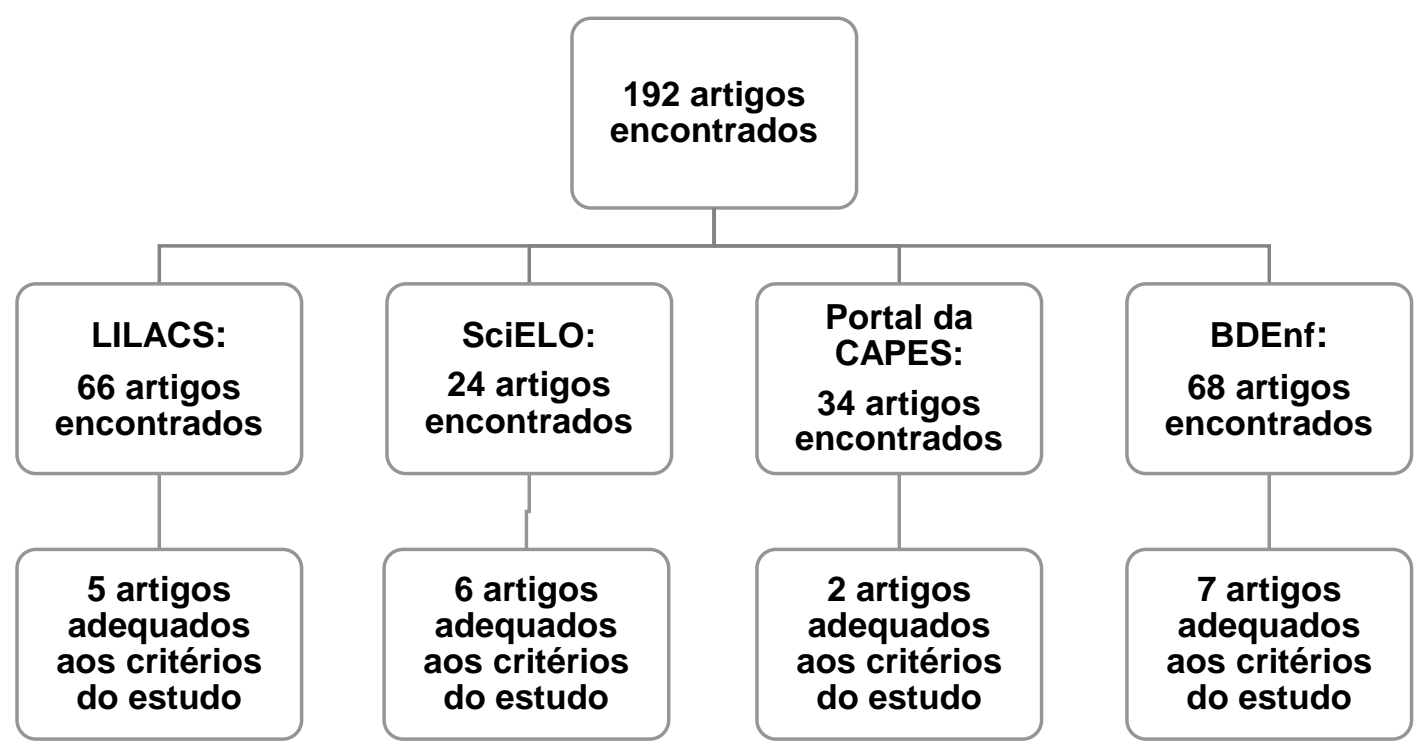

Fonte: Nascimento MT, Silva NIO, Brito FCBA, et al., 2019.

\section{RESULTADOS E DISCUSSÃO}

Após análise dos artigos selecionados foram levantadas duas categorias para discussão: "Dificuldades de autocuidado e fatores de risco que favorecem o desenvolvimento do pé diabético" e "Condutas prevencionistas do enfermeiro da Atenção Primária à Saúde para impedir o surgimento do pé diabético".

\section{Dificuldades de autocuidado e fatores de risco que favorecem o desenvolvimento do pé diabético}

O conhecimento de diabéticos acerca das ações de autocuidado adequadas com os membros inferiores é essencial para superação de condutas errôneas, para diminuição de alterações fisiopatológicas que contribuem ao desenvolvimento de úlceras nos pés e para promoção do protagonismo do sujeito diabético no auto manejo de sua doença (POLICARPO NS et al., 2014).

O estudo de Rezende Neta DS et al. (2015) realizado com 331 participantes diabéticos tipo II mostrou que cerca de $54 \%$ dos sujeitos nunca receberam orientações do enfermeiro sobre a necessidade de avaliação dos pés e de secagem dos espaços interdigitais diariamente, do mesmo modo que 66,5\% negaram maiores instruções quanto à inspeção de calçados. Faz parte das ações do enfermeiro durante a consulta de 
Enfermagem ao diabético educá-lo a cuidados que devem dispor com seus pés, tais como inspeção diária, higiene e enxugamento, principalmente entre os dedos. Na oportunidade, também é relevante o estímulo ao uso de sapatos fechados e confortáveis, assim como exame minucioso antes de calcá-los.

A baixa escolaridade foi notada em diferentes estudos selecionados para composição desta revisão (TAVARES VS et al., 2014; SILVA LWS et al., 2016). Por vezes, a baixa escolaridade é um fator que eleva as chances de desenvolvimento do pé diabético, isso porque o indivíduo com pouco tempo de estudo possui dificuldades de entendimento das orientações ofertadas pelos profissionais de saúde e não consegue aderir às práticas de autocuidado (CARLESSO GP et al., 2017).

A não execução ou realização das ações de autocuidado de forma inadequada traz consequências à saúde do diabético e denota déficit de conhecimento prévio, que pode estar associado à restrição no acesso a informações que devem ser dadas pelo profissional atuante na APS, conforme revelam os estudos de Policarpo NS et al. (2014) e Menezes LCG et al. (2017) ao constatarem que os participantes das respectivas pesquisas buscam instituir cuidados com os próprios pés, contudo de maneira incompleta, como a não secagem adequada dos pés, o tipo de corte das unhas e a ferramenta utilizada (tesoura com ponta).

O estudo de Rossaneis MA et al. (2016) realizado 1.515 sujeitos objetivou verificar distinções no autocuidado com os pés entre mulheres e homens diabéticos. Nele, constatou-se a inexistência de um padrão de cuidados conforme o sexo, pois ao passo em que mulheres apresentaram elevada prevalência de cuidados voltados à prevenção de lesões traumáticas, os homens manifestaram melhores hábitos no uso de calçados.

Associado à baixa escolaridade, o poder aquisitivo limitado é outro fator preponderante, pois impede condições necessárias ao integral tratamento da DM, como a ingesta de alimentos saudáveis e variabilidade do cardápio, acesso aos serviços de saúde, fármacos e terapias não disponibilizadas pelo Sistema Único de Saúde, e levando em conta o risco de pé diabético, a compra de calçados apropriados para prevenção de lesões (OLIVERA NETO M et al., 2017).

O uso de calçados adequados em sujeitos que apresentam diminuição da sensibilidade plantar colabora para reduzir o cisalhamento, a pressão e a absorção do impacto de traumas. O acabamento de sapatos terapêuticos para pacientes diabéticos é útil na prevenção de complicações (FASSINA G et al., 2018).

O tempo decorrido até o diagnóstico da DM também se apresenta como aspecto importante no surgimento de complicações nos pés, tendo o período superior a 10 anos como fator de risco adicional para o desenvolvimento de ulcerações em membros inferiores. Correlacionado a isso, o desconhecimento de comorbidades como a hipertensão arterial sistêmica associada a obesidade, a dislipidemia e a doença arterial coronariana não tratadas, também repercutem para a progressão do pé diabético e outras complicações da DM ao comprometer micro vasos (TAVARES TA et al., 2016).

A prática tabagista foi notadamente inferior em um estudo realizado com 31 diabéticos atendidos na APS da cidade de Diamantina-MG (PAULA DB et al., 2016), o que vai de encontro a outros estudos realizados no Brasil (SILVEIRA DM et al., 2017; TAVARES TA et al., 2016) O incentivo ao afastamento do tabagismo como conduta de prevenção de complicações e manutenção do estilo de vida é pertinente, tendo em vista que modificações ateroscleróticas surgem em pessoas mais jovens diagnosticadas com DM e o ato de fumar constitui fator de risco ao desenvolvimento do pé diabético (PAULA DB et al., 2016).

$\mathrm{Na}$ terapêutica não farmacológica engloba-se as mudanças comportamentais, como a reeducação alimentar, que deve seguir um plano alimentar singular para cada paciente com a prática regular de atividade física, sendo medida crucial e de primeira escolha. A não adesão à terapêutica não farmacológica proposta aparece como outro ponto importante. É consenso na literatura que a aderência à terapia possibilita o manejo do peso corporal, afastando assim a obesidade e melhorando o controle glicêmico e indiretamente contribuindo para a profilaxia de diversos agravos da DM (LIMA IG et al., 2017).

Listou-se nesta categoria as principais dificuldades de autocuidado e os fatores de risco relacionados ao surgimento de ulcerações nos pés. Salienta-se algo já pautado na discussão: a importância do conhecimento para prevenção de complicações da DM. Para que o diabético incorpore orientações profissionais no seu dia 
a dia é necessário estreitamento de vínculo com sua patologia, de modo que ele compreenda as condutas que devem ser adotadas. Sugere-se, portanto, a realização de atividades educativas e prestação de uma assistência singular direcionada a cada sujeito, utilizando mecanismos da problematização a fim de facilitar a adesão às práticas de autocuidado.

\section{Condutas prevencionistas do enfermeiro da Atenção Primária à Saúde para impedir o surgimento do pé diabético}

O enfermeiro exerce função importante na prevenção do pé diabético. Isso porque suas ações de educação podem potencializar o cuidado e auxiliar na detecção precoce de modificações na sensibilidade da pele e dos membros inferiores. O trabalho desempenhado por este profissional, se bem executado, aumenta a adesão do paciente ao tratamento e possibilita maiores êxitos terapêuticos (SILVEIRA DM et al., 2017).

É de competência do enfermeiro, no âmbito da APS, a avaliação clínica do diabético, que engloba: exame minucioso dos pés e de lesões, acompanhamento rotineiro e oferecimento de informações aos indivíduos e familiares quanto a importância de cuidado com os pés. Essas ações são executadas dentro da consulta de Enfermagem (PEREIRA LF et al., 2017).

A consulta juntamente com a prescrição de cuidados de Enfermagem é uma atividade específica do enfermeiro, indispensável para o reconhecimento de riscos à saúde. Ao identificar problemas potenciais esse profissional encaminha seu cuidado para a tomada de decisões. A consulta oportuniza ao enfermeiro o planejamento de sua assistência respeitando a singularidade de cada paciente atendido, proporcionando um atendimento eficaz e resolutivo (VARGAS CP et al., 2017).

Por meio do exame físico realizado na consulta de Enfermagem, verifica-se o risco de o paciente evoluir com o desenvolvimento do pé diabético. Esse momento é essencial para definir a quantidade necessária de avaliações e consultas para cada paciente, proporcionando assim atendimento singular, sendo que os indivíduos sem risco devem voltar para reavaliação continuamente a cada seis meses (SILVA JMTS et al., 2017). Para a avaliação clínica do paciente faz-se necessária a adoção de instrumentos que facilitem o trabalho do profissional e o acompanhamento do diabético. O estudo de Melo RFA et al. (2017) propôs a criação de uma ficha de avaliação clínica dos membros inferiores. A ferramenta elaborada pelos autores constitui-se de quatro dimensões: entrevista com dados necessários à anamnese; exame clínico e estabelecimento do risco de evolução do pé diabético; investigação do autocuidado com os pés; e orientações educacionais na prevenção da complicação.

Teston EF et al. (2017) evidencia que a classificação de risco de ulceração nos pés deve ocorrer de acordo com o que preconiza o Ministério da Saúde. Para auxiliar nessa classificação, pode ser aplicada durante o exame físico a escala que avalia os graus de comprometimento dos membros inferiores, conforme segue: Grau 0: neuropatia ausente (sensibilidade presente); Grau 1: neuropatia presente (modificação de sensibilidade); Grau 2: neuropatia presente, indícios de patologia vascular periférica elou deformidade nos membros inferiores; Grau 3: antecedentes de amputação.

Outra estratégia empregada na avaliação da sensibilidade dos pés é o teste do monofilamento. Este é recurso de baixo custo que possui um fio de nylon apoiado em uma haste com peso de dez gramas, o qual é aplicado perpendicularmente na região do pé a um ângulo de $90^{\circ}$ por meio do sistema de resposta "sim-não" ao toque do aparelho. $O$ fio deve ser aplicado com força suficiente para este se encurvar e o tempo de contato não deve ultrapassar dois segundos em cada região pesquisada (REZENDE NETA DS et al., 2015).

É recomendado que quatro regiões sejam pesquisadas no teste de monofilamento: hálux e o primeiro, o terceiro e o quinto limite dos metatarsos de cada pé. O exame possui sensibilidade de $90 \%$ e especificidade de $80 \%$. A redução ou insensibilidade empregando a pressão de monofilamento é extremamente preditiva de ulcerações futuras (TAVARES TA et al., 2016).

As condutas desenvolvidas pelo enfermeiro na prevenção do pé diabético não devem limitar-se apenas à consulta de Enfermagem. Práticas de educação em saúde também devem ser executadas, pois promovem a sensibilização dos sujeitos para atos de autocuidado (PEREIRA LF et al., 2017). 
A educação em saúde pautada em comunicação dialógica e relacionamento horizontal e prolongado reduz as complicações ocasionadas pelo adoecimento ao passo em que favorece a autonomia no caminho do autocuidado (SILVA LWS et al., 2016b). Tais ações educativas podem ocorrer dentro da própria consulta ou por meio de rodas de conversa, palestras ou discussão em grupo (GOMES DM et al., 2017).

Nesta categoria discutiu-se as condutas do enfermeiro na prevenção do pé diabético no âmbito da APS. Percebeu-se que, na literatura, predominam ações preventivas voltadas à educação do paciente, seja por meio de orientações durante as consultas ou por atividades educativas específicas para grupos. Essas ações de educação são essenciais para aquisição de conhecimento, contribuem para superação de hábitos inadequados de diabéticos e propiciam a redução das complicações.

\section{CONSIDERAÇÕES FINAIS}

As estratégias de prevenção do pé diabético no âmbito da Atenção Primária à Saúde foram diversas, mas percebe-se condutas direcionadas principalmente a orientações com incentivo à obtenção de conhecimento acerca do processo saúde-doença. Compreende-se, portanto, que o entendimento sobre o estado de saúde empodera o diabético e estimula seu protagonismo e conscientização na adesão às práticas de autocuidado. As limitações deste estudo relacionam-se a determinados aspectos estabelecidos metodologicamente, como a língua adotada ser unicamente a portuguesa. Contudo, suas contribuições referem-se à identificação dos obstáculos de autocuidado enfrentados pelo paciente, bem como dos principais fatores de risco ao desenvolvimento do pé diabético. Conhecer tais fatores possibilita a implementação de ações profiláticas pelo enfermeiro e demais profissionais da Atenção Primária à Saúde. Contribui também ao salientar o papel prevencionista do enfermeiro na detecção precoce daqueles diabéticos com maiores risco de evolução com a complicação.

\section{REFERÊNCIAS}

1. BRASIL. Ministério da Saúde. Secretaria de Atenção à Saúde. Departamento de Atenção Básica. Manual do pé diabético: estratégias para o cuidado da pessoa com doença crônica. Brasília: Ministério da Saúde, 2016.

2. CARLESSO GP et al. Avaliação do conhecimento de pacientes diabéticos sobre medidas preventivas do pé diabético em Maringá (PR). Jornal Vascular Brasileiro, 2017; 16(2): 113-118.

3. ERCOLE FF et al. Revisão integrativa versus revisão sistemática. Revista Mineira de Enfermagem, 2014; 18(1): 911.

4. FASSINA G et al. Avaliação do autocuidado em pacientes portadores do pé diabético. Revista da Faculdade de Ciências Médicas de Sorocaba, 2018; 20(4): 200-206.

5. GOMES DM et al. Ressignificação do cuidado de uma pessoa com diabetes e pé diabético: relato de experiência. Revista de Enfermagem do Centro-Oeste Mineiro, 2018; 8(1): e1509.

6. IMAZU MFM et al. Efetividade das intervenções individual e em grupo junto a pessoas com diabetes tipo 2 . Revista Latino-Americana de Enfermagem, 2015; 23(2): 200-207.

7. LIMA IG et al. Educar para prevenir: a importância da informação no cuidado do pé diabético. Revista Conexão UEPG, $2017 ; 13(1):$ 186-195.

8. MELO RFA et al. Ficha de avaliação clínica de membros inferiores para prevenção do pé diabético. Revista de Pesquisa: Cuidado é Fundamental, 2017; 9(3): 899-913.

9. MENEZES LCG et al. Pesquisa ação: práticas de autocuidado das pessoas com pé diabético. Revista de Enfermagem da UFPE, 2017; 11(S9): 3558-3566.

10. OLIVEIRA PS et al. Atuação dos enfermeiros da estratégia saúde da família na prevenção do pé diabético. Revista de Pesquisa: Cuidado é Fundamental, 2016; 8(3): 4841-4849.

11. OLIVEIRA NETO $\mathrm{M}$ et al. Avaliação do autocuidado para a prevenção do pé diabético e exame clínico dos pés em um centro de referência em diabetes mellitus. Journal of Health \& Biological Sciences, 2017; 5(3): 265-271.

12. PAULA DB et al. Avaliação dos pés em indivíduos portadores de diabetes atendidos em uma unidade de atenção primária. Revista de Enfermagem da UFPE, 2016; 10(S6): 4751-4756.

13. PEREIRA LF et al. Ações do enfermeiro na prevenção do pé diabético: o olhar da pessoa com diabetes mellitus. Revista de Pesquisa: Cuidado é Fundamental, 2017; 9(4): 1008-1014. 
14. PETERMANN XB et al. Epidemiologia e cuidado à Diabetes Mellitus praticado na Atenção Primária à Saúde: uma revisão narrativa. Revista Saúde, 2015; 41(1): 49-56.

15. POLICARPO NS et al. Conhecimento, atitudes e práticas de medidas preventivas sobre pé diabético. Revista Gaúcha de Enfermagem, 2014; 35(3): 36-42.

16. REZENDE NETA DS et al. Adesão das pessoas com diabetes mellitus ao autocuidado com os pés. Revista Brasileira de Enfermagem, 2015; 68(1): 111-116.

17. ROSSANEIS MA et al. Diferenças entre mulheres e homens diabéticos no autocuidado com os pés e estilo de vida. Revista Latino-Americana de Enfermagem, 2016; 24(1): e2761.

18. SILVA CAM et al. Pé diabético e avaliação do risco de ulceração. Revista Enfermagem Referência, 2014; 4(5): 153161.

19. SILVA JMTS et al. Fatores associados à ulceração nos pés de pessoas com diabetes mellitus residentes em área rural. Revista Gaúcha de Enfermagem, 2017; 38(3): e68767.

20. SILVA LWS et al. Cuidado dos pés de pessoas com diabetes mellitus: ações protetivas vinculadas à promoção da saúde. Enfermería: Cuidados Humanizados, 2016; 5(2): 12-18, 2016.

21. SILVEIRA DM et al. Pé Diabético: onde podemos intervir? HU Revista, 2017; 43(1): 13-18.

22. SMANIOTO FN et al. Autocuidado nos fatores de risco na ulceração do pé diabético: estudo transversal. Online Brazilian Journal of Nursing, 2014; 13(3): 343-352.

23. SOARES CB et al. Revisão integrativa: conceitos e métodos utilizados na enfermagem. Revista Escola de Enfermagem da USP, 2014; 48(2): 335-345.

24. TAVARES TA et al. Fatores de risco para ulceração e amputação de extremidades inferiores em portadores de diabetes mellitus. Revista Brasileira em Promoção da Saúde, 2016; 29(2): 278-287.

25. TAVARES VS et al. Avaliação da atenção ao diabetes mellitus em Unidades de Saúde da Família de Petrolina, Pernambuco, 2011. Revista Epidemiologia e Serviços de Saúde, 2014; 23(3): 527-536.

26. TESTON EF et al. Fatores de risco para ulceração no pé de indivíduos com diabetes mellitus tipo 2. Cogitare Enfermagem, 2017; 22(4): e51508.

27. VARGAS CP et al. Condutas dos enfermeiros da atenção primária no cuidado a pessoas com pé diabético. Revista de Enfermagem da UFPE, 2017; 11(S11): 4535-4545. 